ISSN 1112-9867

Available online at

http://www.jfas.info

\title{
MAXIMUM LIKELIHOOD ESTIMATION OF EXPONENTIAL DISTRIBUTION UNDER TYPE-II CENSORING FROM IMPRECISE DATA
}

\author{
I. Makhdoom* and P. Nasiri
}

Department of Statistics, Payame Noor University(PNU), P.O.BOX, 19395-3697, Tehran,Iran

Published online: 14 June 2016

\begin{abstract}
Statistical analysis of lifetime distributions under Type-II censoring scheme is based on precise lifetime data. However, some collected lifetime data might be imprecise and are represented in the form of fuzzy numbers. This paper deals with the estimation of exponential mean parameter under Type-II censoring scheme when the lifetime observations are fuzzy and are assumed to be related to underlying crisp realization of a random sample. Maximum likelihood estimate of the unknown parameter is obtained by using EM algorithm. In addition, a new numerical method for parameter estimation is provided. Using the parametric bootstrap method, the construction of confidence intervals for the mean parameter is discussed. Monte Carlo simulations are performed to investigate performance of the different methods. Finally, an illustrative example is also included.
\end{abstract}

Keywords: Type-II censoring, Imprecise lifetime, Exponential distribution, Maximum likelihood estimation, Bootstrap confidence interval

Author Correspondence, e-mail: makhdoom@pnu.ac.ir

doi: http://dx.doi.org/10.4314/jfas.8vi2s.42

\section{INTRODUCTION}

In many life testing experiments, the experimenter may not observe the lifetimes of all inspected units in the life test. This may be because of time limitation and/or other restrictions (such as money and material resources, etc) on data collection. Censored data arises in these 
situations wherein the experimenter does not obtain complete information for all the units under study. Different types of censoring arise based on how the data are collected from the life-testing experiment. The scheme of type-II censored sampling is an important method of obtaining data in life testing experiments. Under this censoring scheme the life testing experiment will be terminated upon the $\mathrm{r}$-th ( $\mathrm{r}$ is pre-fixed) failure. This scheme is often adopted for toxicology experiments and life testing applications by engineers as it has been proven to save time and money. Several authors have addressed inferential issues based on Type-II censored samples; see for example, [24], [23], [3], [8], [2], [6], [25], [18], [4], [5], [16], [22]. Their research results for estimating the parameters of lifetime different distributions under Type-II censoring are based on precise lifetime data. However, in real situations all observations and measurements of continuous variables are not precise numbers but more or less non-precise. This imprecision is different from variability and errors. Therefore also lifetime data are not precise numbers but more or less fuzzy. The best up-to-date mathematical model for this imprecision are so-called non-precise numbers.

Example 1. Assume that $n$ identical ball bearings are placed on a life-testing experiment, and we are interested in the lifetime of these ball bearings. In practice, however, measuring the lifetime of a ball bearing may not yield an exact result. A ball bearing may work perfectly over a certain period but be braking for some time, and finally be unusable at a certain time. So, the number of revolutions to failure (in millions) for ball bearings may reported by means of the following imprecise quantities: " approximately lower than 45 ”, “ approximately 50 to 70 ”, “ approximately 75 ”, “ approximately 80 ”, “ approximately 90 to 100 ”, “ approximately higher than 120 ", and so on.

Classical statistical procedures and Bayesian inference are not appropriate to deal with such imprecise cases. Therefore, we need suitable statistical methodology to handle these data as well. In recent years, several researchers pay attention to applying the fuzzy sets to estimation theory. Huang et al. [15] proposed a new method to determine the membership function of the estimates of the parameters and the reliability function of multiparameter lifetime distributions. Coppi et al. [7] presented some applications of fuzzy techniques in statistical analysis. Pak et al. 
([19],[20] and [21] ) conducted a series of studies to develop the inferential procedures for the lifetime distributions on the basis of fuzzy data.

In this paper, our objective is to devise the methods for parameter estimation regarding a life-test from which the Type-II censored data are reported in the form of fuzzy numbers. We analyze the data under the assumptions that the lifetimes of the test units are independent identically distributed exponential random variables. In Section 2, we first present in greater detail the problem addressed in this paper. Some preliminary concepts about fuzzy numbers is included in this section. In Section 3, we propose a procedure to determine the maximum likelihood estimate (MLE) of the parameter of interest. A new parameter estimation method, called 'computational approach estimation' (CAE), is established in Section 4. By using the parametric bootstrap method, construction of the confidence intervals for the unknown parameter is discussed in Section 5. Simulation study will be carried out to assess the performance of the different proposed methods in section 6. A practical example in life testing is provided in Section 7 in order to illustrate all the methods of inference discussed here.

\section{PROBLEM DESCRIPTION}

Consider a reliability experiment in which $n$ identical units are placed on a life-test. Let $X_{1}, \ldots, X_{n}$ denote the lifetimes of these experimental units. We assume that these variables are independent and identically distributed as Exponential $E(\theta)$, with probability density function (pdf),

$$
f(x ; \theta)=\frac{1}{\theta} \exp \left(-\frac{x}{\theta}\right) \quad, x>0, \theta>0 .
$$

Let $X_{1: n} \leq X_{2: n} \leq \ldots \leq X_{n: n}$ denote the corresponding ordered lifetimes. Suppose the experimenter decides to carry out the life-test until the time of the $r$ th failure, then the data arising from such a life-test would be of the form $X_{1: n} \leq X_{2: n} \leq \ldots \leq X_{r: n}$ with the remaining $n-r$ lifetimes being more than $X_{r: n}$. This situation is referred to as Type-II censoring. We 
also denote the observed values of such a Type-II right censored sample by $x_{1: n}, \ldots, x_{r: n}$. Based on these observations, the likelihood function is given by:

$$
L(\theta ; \mathbf{x})=\frac{n !}{(n-r) ! \theta^{r}} \exp \left(-\frac{\sum_{i=1}^{r} x_{i: n}+(n-r) x_{r: n}}{\theta}\right) .
$$

Thus the maximum likelihood estimator of $\theta$ can be obtained by

$$
\hat{\theta}=\frac{1}{r}\left(\sum_{i=1}^{r} x_{i: n}+(n-r) x_{r: n}\right) .
$$

In many fields of application it is sometimes impossible to obtain exact observations of lifetime. The obtained lifetime data may be imprecise most of the time. For example, the lifetime of units may be between 1500 and $2000 h$, but near to $2000 h$. In order to model these observed lifetimes, a generalization of real numbers is necessary. These lifetimes can be represented by fuzzy numbers. A fuzzy number is a subset, denoted by $\tilde{x}$, of the set of real numbers (denoted by $R$ ) and is characterized by the so called membership function $\mu_{\tilde{x}}($.$) .$ Fuzzy numbers satisfy the following constraints ([10]):

(1) $\mu_{\tilde{x}}: R \rightarrow[0,1]$ is Borel-measurable;

(2) $\exists x_{0} \in \mathrm{R}: \mu_{\tilde{x}}\left(x_{0}\right)=1$;

(3) The so-called $\lambda$-cuts $(0<\lambda \leq 1)$, defined as $B_{\lambda}(\tilde{x})=\left\{x \in R: \mu_{\tilde{x}}(x) \geq \lambda\right\}$, are all closed interval, i.e., $B_{\lambda}(\tilde{x})=\left[a_{\lambda}, b_{\lambda}\right], \forall \lambda \in(0,1]$.

With the definition of a fuzzy number given above, an exact (non-fuzzy) number can be treated as a special case of a fuzzy number. For a non-fuzzy real observation $x_{0} \in \mathrm{R}$, its corresponding membership function is $\mu_{x_{0}}\left(x_{0}\right)=1$. Usually, $L R$-type fuzzy numbers (the triangular and trapezoidal fuzzy numbers are special cases of the $L R$-type fuzzy numbers) are most convenient and useful in describing fuzzy lifetime observations. Therefore, we shall focus on the set of $L R$-type fuzzy numbers.

Definition 1 ([27] pp.62). Let $L$ (and $R$ ) be decreasing, shape functions from $\mathrm{R}^{+}$to $[0,1]$ with $L(0)=1 ; L(x)<1$ for all $x>0 ; L(x)>0$ for all $x<1 ; L(1)=0$ or $(L(x)>0$ for all 
$x$ and $L(+\infty)=0$ ). Then a fuzzy number $\tilde{x}$ is called of $L R$-type if for $m, \alpha>0, \beta>0$ in $\mathrm{R}$,

$$
\mu_{\tilde{x}}(x)= \begin{cases}L\left(\frac{m-x}{\alpha}\right) & x \leq m \\ R\left(\frac{x-m}{\beta}\right) & x \geq m\end{cases}
$$

where $m$ is called the mean value of $\tilde{x}$ and $\alpha$ and $\beta$ are called the left and right spreads, respectively. Symbolically, the $L R$-type fuzzy number is denoted by $\tilde{x}=(\alpha, m, \beta)$.

Definition 2 Suppose that $\tilde{x}_{i}=\left(\alpha_{i}, m_{i}, \beta_{i}\right), i=1, \ldots, n$, be the $L R$-type fuzzy numbers. The fuzzy mean value of these numbers can be obtained as

$$
" \bar{x}=(\bar{\alpha}, \bar{m}, \bar{\beta}) \text {, with } \bar{\alpha}=\frac{1}{n} \sum_{i=1}^{n} \alpha_{i}, \bar{m}=\frac{1}{n} \sum_{i=1}^{n} m_{i} \text { and } \bar{\beta}=\frac{1}{n} \sum_{i=1}^{n} \beta_{i} .
$$

Our viewpoint in this paper is based on an epistemic interpretation of fuzzy data, which are assumed to "imperfectly specify a value that is existing and precise, but not measurable with exactitude under the given observation conditions" ([13], p. 316). In this model, a fuzzy datum is thus seen as a possibility distribution associated to a precise realization of a random variable that has been only partially observed. In the next section, we introduce a generalization of the likelihood function and obtain the maximum likelihood estimate (MLE) of the unknown parameter $\theta$.

\section{MAXIMUM LIKELIHOOD ESTIMATION}

Now consider the problem where under the Type-II censoring scheme, failure times are not observed precisely and only partial information about them are available in the form of fuzzy numbers $\tilde{x}_{i}=\left(\alpha_{i}, m_{i}, \beta_{i}\right), i=1, \ldots, r$, with their corresponding membership functions $\mu_{\tilde{x}_{1}}\left(x_{1}\right), \ldots, \mu_{\tilde{x}_{r}}\left(x_{r}\right)$. Let the maximum value of the means of these fuzzy numbers to be $m_{(r)}$. The lifetime of $n-r$ surviving units can be encoded as fuzzy numbers $\tilde{x}_{r+1}, \ldots, \tilde{x}_{n}$ with the membership functions 


$$
\mu_{\tilde{x}_{i}}\left(x_{i}\right)= \begin{cases}0 & x \leq m_{(r)} \\ 1 & x>m_{(r)}\end{cases}
$$

for $i=r+1, \ldots, n$. The fuzzy data $\tilde{\mathbf{x}}=\left(\tilde{x}_{1}, \ldots, \tilde{x}_{n}\right)$ is thus the vector of observed lifetimes. The corresponding observed-data log-likelihood function can be obtained, using Zadeh's definition of the probability of a fuzzy event ([26]), as

$$
\begin{aligned}
& L_{c}(\theta ; \tilde{\mathbf{x}})=\sum_{i=1}^{n} \log \int \frac{1}{\theta} \exp \left(-\frac{x}{\theta}\right) \mu_{\tilde{x}_{i}}(x) d x \\
& =\sum_{i=1}^{r} \log \int \frac{1}{\theta} \exp \left(-\frac{x}{\theta}\right) \mu_{\tilde{x}_{i}}(x) d x+(n-r) \log \left\{\int_{m_{(r)}}^{\infty} \frac{1}{\theta} \exp \left(-\frac{x}{\theta}\right) d x\right\} .
\end{aligned}
$$

Since the observed fuzzy data $\tilde{\mathbf{x}}$ can be seen as an incomplete specification of a complete data vector $\mathbf{x}$, the EM algorithm can be applied to maximize the observed-data log-likelihood (3). Therefore, in the following we use the fuzzy EM (FEM) algorithm ([9]) to determine the MLE of $\theta$. Each iteration of the algorithm involves two steps called the expectation step (E-step) and the maximization step (M-step). The E-step requires the calculation of

$$
E\left(\log L(\theta ; \mathbf{X}) \mid \tilde{\mathbf{x}} ; \theta^{(h)}\right)=-n \log (\theta)-\frac{1}{\theta} \sum_{i=1}^{n} E\left(X_{i} \mid \tilde{x}_{i} ; \theta^{(h)}\right)
$$

in which $L(\theta ; \mathbf{X})$ is the complete-data likelihood function and $\theta^{(h)}$ denotes the current fit of $\theta$ at iteration $h$. The conditional expectations $E\left(X_{i} \mid \tilde{x}_{i} ; \theta^{(h)}\right), i=1, \ldots, n$, can be computed using:

$$
E\left(X \mid \tilde{x} ; \theta^{(h)}\right)=\int x g\left(x \mid \tilde{x} ; \theta^{(h)}\right) d x .
$$

where the conditional density of $X$ given $\tilde{x}$ is given by

$$
g\left(x \mid \tilde{x} ; \theta^{(h)}\right)=\frac{1}{\theta^{(h)} E\left(\mu_{\tilde{x}}(X)\right)} \exp \left(-\frac{x}{\theta^{(h)}}\right) \mu_{\tilde{x}}(x)
$$

The M-step then consists in finding $\theta^{(h+1)}$ which maximizes $E\left(\log L(\theta ; \mathbf{x}) \mid \widetilde{\mathbf{x}} ; \theta^{(h)}\right)$. This is easily achieved by solving the likelihood equation. From

$$
\frac{\partial}{\partial \theta} E\left(\log L(\theta ; \mathbf{X}) \mid \tilde{\mathbf{x}} ; \theta^{(h)}\right)=0
$$


we get

$$
\theta^{(h+1)}=\frac{1}{n} \sum_{i=1}^{n} E\left(X_{i} \mid \tilde{x}_{i} ; \theta^{(h)}\right)
$$

The MLE of $\theta$ can be obtained by repeating the E- and M-steps until the difference $L_{c}\left(\theta^{(h+1)} ; \tilde{\mathbf{x}}\right)-L_{c}\left(\theta^{(h)} ; \widetilde{\mathbf{x}}\right)$ becomes smaller than some arbitrary small amount.

It is showed in ([9]) that the observed-data log-likelihood $L_{c}(\theta ; \widetilde{\mathbf{x}})$ is not decreased after an EM iteration. Hence, convergence to some value $L^{*}$ is ensured as long as the sequence $L_{c}\left(\boldsymbol{\theta}^{(h)} ; \tilde{\mathbf{x}}\right)$ for $h=0,1, \ldots$ is bounded from above.

\section{COMPUTATIONAL APPROACH ESTIMATION METHOD}

In this section we propose a new parameter estimation procedure called 'CAE'. Although the maximum likelihood estimate obtained in the preceding section is preferable, its computation requires repeated evaluation of $\mathrm{E}$ - and $\mathrm{M}$-steps until convergence occurs. On the other hand, the CAE method provides not only the computational ease but also reasonable mean squared error. This finding is further discussed in Section 6. Suppose $\tilde{x}_{i}=\left(\alpha_{i}, m_{i}, \beta_{i}\right), i=1, \ldots, r$, be the observed fuzzy lifetimes under Type-II censoring from exponential distribution with unknown parameter $\theta$. Grzegorzewski and Hryniewicz [14] considered the generalization of exponential model which admits vagueness in lifetimes. They obtained a fuzzy estimator of the mean lifetime $\theta$ in the presence of vague lifetimes. However, in most applications, crisp results are required instead of fuzzy ones. So, we propose the following computational approach to obtain a crisp value as an estimate of $\theta$.

Step 1: Order the means of fuzzy numbers as $m_{(1)}<m_{(2)}<\ldots<m_{(r)}$.

Step 2: Obtain the fuzzy mean value, say $x$, of the fuzzy numbers by using (2).

Step 3: Convert the fuzzy number $x$ into a real value by using the center of gravity defuzzification technique. Denote this defuzzified value by $\underset{x}{*}$.

Step 4: The new estimate of $\theta$ then is computed as: 


$$
\tilde{\tilde{\theta}}=\tilde{\tilde{x}}^{*}+\left(\frac{n}{r}-1\right) m_{(r)}
$$

\section{BOOTSTRAP CONFIDENCE INTERVAL}

In this section, we discuss the construction of the confidence interval (CI) for the unknown parameter $\theta$ using the two types of bootstrap methods, viz., percentile bootstrap (Boot-p) method and the bias-corrected and accelerated (BCa) percentile bootstrap method; see [11] and [12] for pertinent details.

Suppose $n$ identical items are put on a test and in the presence of Type-II censoring scheme, the observed lifetimes are reported as fuzzy numbers $\tilde{x}_{i}=\left(\alpha_{i}, m_{i}, \beta_{i}\right), i=1, \ldots, r$. Before we discuss the construction of the bootstrap confidence intervals for $\theta$, the following algorithm is used to generate the bootstrap sample of fuzzy numbers based on the original Type-II censored fuzzy sample $\tilde{x}_{1}, \ldots, \tilde{x}_{r}$.

Step 1: Given the original Type-II censored fuzzy sample $\tilde{x}_{1}, \ldots, \tilde{x}_{r}$, compute the MLE of $\theta$, say $\hat{\theta}$, using the iterative algorithm (8).

Step 2: Generate the Type-II censored sample of size $r$, say $m_{1}^{*}, \ldots, m_{r}^{*}$, with the underlying distribution as $E(\hat{\theta})$. Define the $L R$ - type fuzzy numbers $\tilde{x}_{1}^{*}, \ldots, \tilde{x}_{r}^{*}$ as $\tilde{x}_{i}^{*}=\left(\alpha_{i}, m_{i}^{*}, \beta_{i}\right), i=1, \ldots, r$.

Step 3: Based on the simulated Type-II censored fuzzy sample in Step 2 ,Calculate the bootstrap MLE of $\theta$, denoted by $\hat{\theta}^{*}$, from (8).

Step 4: Repeat step 2 and 3, M times. Then, arrange all bootstrapped values of $\hat{\theta}^{*}$ in ascending order to obtain the ordered bootstrap sample of $\hat{\theta}_{1}^{*}<\hat{\theta}_{2}^{*}<\ldots<\hat{\theta}_{M}^{*}$.

With the bootstrap sample generated as above, we propose the following two parametric bootstrap confidence interval for $\theta$.

\section{Boot-p confidence interval:}

A two sided $100(1-\gamma) \%$ percentile bootstrap CI for $\theta$ is

$$
\left(\hat{\theta}_{\left[M\left(\frac{\gamma}{2}\right)\right]}^{*}, \hat{\theta}_{\left[M\left(1-\frac{\gamma}{2}\right)\right]}^{*}\right)
$$




\section{BCa percentile bootstrap confidence interval:}

A two sided $100(1-\gamma) \%$ BCa percentile bootstrap CI for $\theta$ is

$$
\left(\hat{\theta}_{[\alpha M]}^{*}, \hat{\theta}_{[\beta M]}^{*}\right),
$$

where

$$
\alpha=\Phi\left(\hat{z}_{0}+\frac{\hat{z}_{0}-z_{\alpha / 2}}{1-\hat{a}\left(\hat{z}_{0}-z_{\alpha / 2}\right)}\right)
$$

and

$$
\beta=\Phi\left(\hat{z}_{0}+\frac{\hat{z}_{0}+z_{\alpha / 2}}{1-\hat{a}\left(\hat{z}_{0}+z_{\alpha / 2}\right)}\right) .
$$

Here, $\Phi($.$) denotes the CDF of the standard normal distribution, z_{\alpha}$ is the upper $\alpha$-point of the standard normal distribution and $[x]$ denote the integer part of $x$. The value of bias-correction $\hat{z}_{0}$ is given by

$$
\hat{z}_{0}=\Phi^{-1}\left(\frac{\sum_{j=1}^{M} I\left(\hat{\theta}_{j}^{*}<\hat{\theta}\right)}{M}\right),
$$

and a good estimate of the acceleration factor $\hat{a}$ is suggested to be

$$
\hat{a}=\frac{\sum_{j=1}^{r}\left(\hat{\theta}^{(.)}-\hat{\theta}^{(j)}\right)^{3}}{6\left\{\sum_{j=1}^{r}\left(\hat{\theta}^{(.)}-\hat{\theta}^{(j)}\right)^{2}\right\}^{3 / 2}},
$$

where $\hat{\theta}^{(j)}$ is the MLE of $\theta$ based on the original Type-II censored fuzzy sample with the jth observation deleted for $j=1, \ldots, r$, and

$$
\hat{\theta}^{(\cdot)}=\frac{1}{r} \sum_{j=1}^{r} \hat{\theta}^{(j)}
$$

\section{SIMULATION STUDY}

In order to evaluate the performance of all the different methods discussed in the preceding sections, a Monte Carlo simulation study was conducted and its results are described in this 
section. First, for different choices of $n, r$ and $\theta$ we generate the Type-II censored sample $x_{1}, \ldots, x_{r}$ from exponential distribution. Then we define fuzzy numbers $\tilde{x}_{1}, \ldots, \tilde{x}_{r}$ with the corresponding membership functions

$$
\mu_{\tilde{x}_{i}}(x)=\left\{\begin{array}{ll}
\frac{x-\left(x_{i}-h_{i}\right)}{h_{i}} & x_{i}-h_{i} \leq x \leq x_{i} \\
\frac{x_{i}+h_{i}-x}{h_{i}} & x_{i} \leq x \leq x_{i}+h_{i}
\end{array}, \quad i=1, \ldots, r\right.
$$

where $h_{i}=0.05 x_{i}$. This procedure simulates the situation where the observer has only approximate knowledge of the failure times, and can only provide a guess $x_{i}$ and an interval of plausible values $\left[x_{i}-h_{i}, x_{i}+h_{i}\right]$. From these fuzzy numbers, we obtain the MLE of $\theta$, using the iterative algorithm (8). We have used the initial estimate to be $\theta^{(0)}=\frac{1}{r} \sum_{i=1}^{r} x_{i}$ and the iterative process stops when the relative change of the log-likelihood becomes less than $10^{-6}$. We also obtain the estimate of $\theta$ using the CAE method. The average values $(A V)$ and the mean squared errors $(M S E)$ of the estimates based on 1000 replication are presented in Table 1. We also compute the $95 \%$ confidence intervals using the Boot-p and BCa percentile bootstrap methods. The average confidence lengths and the coverage probabilities of the confidence intervals are reported in Table 2.

From Table 1, the following observations are made. The performance of the MLEs are satisfactory in terms of AVs and MSEs. For fixed $n$ as $r$ increases, the MSEs decrease for all cases as expected. Similar observations are made for the estimates of $\theta$ obtained from the CAE method. It is also observed that, the MSEs of the estimates based on the CAE method are quite close to that of the MLEs.

Among the bootstrap methods for constructing confidence interval of $\theta, \mathrm{BCa}$ percentile bootstrap method is better than the Boot-p method with respect to the coverage probabilities. From Table 2, we can see that the coverage probabilities of the BCa confidence intervals are close to the nominal level unless the effective relative sample fraction $\left(\frac{r}{n}\right)$ is small, while the 
same of the Boot-p confidence intervals are lower than the nominal level. The average confidence lengths of the Boot-p confidence intervals are slightly smaller than the BCa confidence intervals. For the two methods it is observed that the average confidence lengths decrease as the effective sample size increases.

\section{ILLUSTRATIVE EXAMPLE}

The following data (the centers of fuzzy numbers, $m_{i}$ ) show the lifetimes (in $1000 \mathrm{~km}$ ) of front disk brake pads on a randomly selected set of 40 cars (same model) that were monitored by a dealer network, ([17], p. 337). But, in practice measuring the lifetime of a disk may not yield an exact result. A disk may work perfectly over a certain period but be braking for some time, and finally be unusable at a certain time. So, such data may be reported as imprecise quantities. Assume that the lifetimes of front disk brake pads are reported as fuzzy numbers given below. In fact, imprecision is formulated by fuzzy numbers $\tilde{x}_{i}=\left(h_{i}, m_{i}\right)$, where $h_{i}=0.005 x_{i}, \quad i=1, \ldots, 40$, with membership functions

$$
\mu_{\tilde{x}_{i}}(x)=\left\{\begin{array}{ll}
\frac{x-\left(m_{i}-h_{i}\right)}{h_{i}} & m_{i}-h_{i} \leq x \leq m_{i} \\
0 & x \geq m_{i}
\end{array} \quad i=1, \ldots, 40 .\right.
$$

DATA SET: $(0.43,86.2),(0.19,38.4),(0.22,45.5),(0.11,22.7),(0.24,48.8)$,

$(0.21,42.8),(0.36,73.1),(0.29,59.8),(0.22,45.1),(0.20,41.0)$

(0.18,36.7), (0.11,22.6), (0.40,81.7), (0.51,102.5), (0.14,28.4),

(0.15,31.7), (0.26,52.1), (0.28,56.4), (0.21,42.2), (0.20,40.0),

(0.30,61.5), (0.21,42.7), (0.23,46.9), (0.16,33.9), (0.27,54.2),

(0.40,81.3), (0.25,51.6), (0.19,38.8), (0.26,53.6), (0.40,80.6),

(0.22,45.9), (0.25,50.6), (0.29,59.0), (0.31,62.4), (0.17,34.4),

(0.25,50.2), (0.25,50.7), (0.32,64.5), (0.16,33.8), (0.28,56.7). 
These data was used by [1] to testing the fuzzy hypothesis for the mean of exponential distribution. From the given data, a Type-II censored sample of size $m=24$ is thus:

$$
\begin{aligned}
& (0.11,22.6),(0.11,22.7),(0.14,28.4),(0.15,31.7), \\
& (0.16,33.8),(0.16,33.9),(0.17,34.4),(0.18,36.7), \\
& (0.19,38.4),(0.19,38.8),(0.20,40.0),(0.20,41.0), \\
& (0.21,42.2),(0.21,42.7),(0.21,42.8),(0.22,45.1), \\
& (0.22,45.5),(0.22,45.9),(0.23,46.9),(0.24,48.8), \\
& (0.25,50.6),(0.25,50.7),(0.25,50.2),(0.25,51.6) .
\end{aligned}
$$

From these data, and using the starting value $\theta^{(0)}=\frac{1}{24} \sum_{i=1}^{24} m_{i}=40.2250$, the final MLE of $\theta$ is found from (8) to be $\hat{\theta}=74.5685$. Fig. 1 shows a plot of the observed-data log-likelihood function as a function of $\theta^{(h)}$. We can check that the MLE corresponds in this case to a global maximum of the observed data log-likelihood. The estimate of $\theta$ from the CAE method becomes $\tilde{\tilde{\theta}}=74.625$.

The $95 \%$ confidence intervals based on Boot-p and BCa methods become

$$
(47.3362,109.3762),(49.7358,112.3634)
$$

respectively.

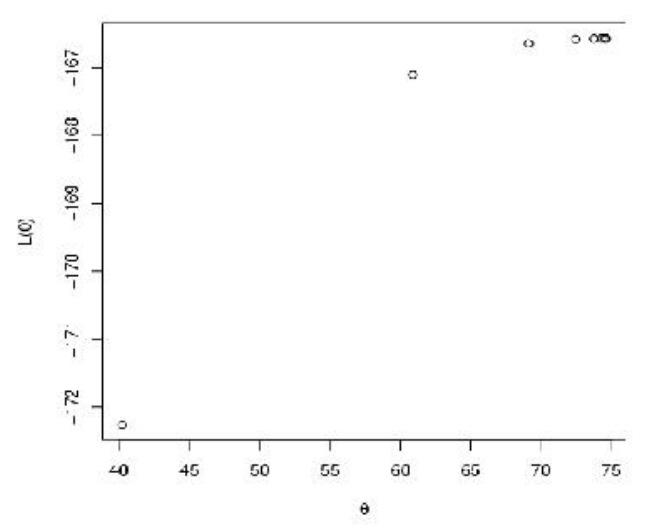

Fig.1. Plot of the observed-data log-likelihood function, as a function of $\theta^{(h)}$ under EM-iterations. 
Table 1. The average values (AV) and the mean squared errors (MSE) of the estimates of $\theta$ based on the ML and the CAE methods.

\begin{tabular}{|c|c|c|c|c|c|c|c|c|c|}
\hline \multirow[t]{3}{*}{$\mathrm{n}$} & \multirow[t]{3}{*}{$\mathrm{r}$} & \multicolumn{4}{|l|}{$\begin{array}{l}\text { cax } \\
0 .\end{array}$} & \multicolumn{4}{|l|}{$\theta=2$} \\
\hline & & \multicolumn{2}{|l|}{ MLE } & \multicolumn{2}{|l|}{ CAE } & \multicolumn{2}{|l|}{ MLE } & \multicolumn{2}{|l|}{ CAE } \\
\hline & & $\mathrm{AV}$ & MSE & $\mathrm{AV}$ & MSE & $\mathrm{AV}$ & MSE & $\mathrm{AV}$ & MSE \\
\hline \multirow[t]{3}{*}{15} & 5 & 0.9857 & 0.2154 & 0.9872 & 0.2170 & 1.9564 & 0.8266 & 1.9617 & 0.8275 \\
\hline & 8 & 1.0123 & 0.1118 & 1.0150 & 0.1121 & 2.0333 & 0.4884 & 2.0348 & 0.4888 \\
\hline & 12 & 0.9998 & 0.0830 & 1.0055 & 0.0832 & 1.9807 & 0.3464 & 1.9837 & 0.3470 \\
\hline \multirow[t]{3}{*}{20} & 5 & 1.0053 & 0.2068 & 1.0081 & 0.2080 & 2.0162 & 0.7226 & 2.0189 & 0.7260 \\
\hline & 10 & 0.9962 & 0.1054 & 1.0075 & 0.1061 & 1.9953 & 0.4010 & 1.9970 & 0.4022 \\
\hline & 15 & 0.9981 & 0.0675 & 0.9939 & 0.0677 & 1.9979 & 0.2742 & 2.0050 & 0.2749 \\
\hline \multirow[t]{3}{*}{30} & 10 & 1.0343 & 0.0953 & 1.0062 & 0.0959 & 1.9808 & 0.3723 & 1.9825 & 0.3742 \\
\hline & 15 & 0.9908 & 0.0670 & 0.9916 & 0.0672 & 1.9826 & 0.2713 & 1.9880 & 0.2729 \\
\hline & 20 & 1.0023 & 0.0527 & 1.0021 & 0.0530 & 1.9962 & 0.2111 & 1.9978 & 0.2113 \\
\hline \multirow[t]{3}{*}{40} & 15 & 1.0032 & 0.0657 & 1.0038 & 0.0657 & 1.9974 & 0.2675 & 2.0073 & 0.2681 \\
\hline & 20 & 0.9992 & 0.0496 & 1.0021 & 0.0497 & 1.9976 & 0.2022 & 1.9927 & 0.2025 \\
\hline & 30 & 0.9992 & 0.0331 & 1.0009 & 0.0331 & 1.9999 & 0.1303 & 1.9987 & 0.1304 \\
\hline \multirow[t]{3}{*}{50} & 15 & 1.0035 & 0.0650 & 1.0051 & 0.0653 & 1.9962 & 0.2586 & 2.0080 & 0.2594 \\
\hline & 20 & 0.9955 & 0.0494 & 0.9956 & 0.0495 & 2.0030 & 0.1811 & 2.0064 & 0.1817 \\
\hline & 30 & 1.0004 & 0.0310 & 1.0006 & 0.0310 & 2.0010 & 0.1212 & 1.9983 & 0.1213 \\
\hline
\end{tabular}


Table 2. The average confidence lengths and the corresponding coverage probabilities of the Boot-p and the BCa confidence intervals for different sample sizes

\begin{tabular}{|c|c|c|c|c|c|c|c|c|c|}
\hline \multirow[t]{4}{*}{$\mathrm{n}$} & \multirow[t]{4}{*}{$\mathrm{r}$} & \multicolumn{4}{|l|}{$=1$} & \multicolumn{4}{|l|}{$o-2$} \\
\hline & & \multicolumn{2}{|l|}{ Boot-p } & \multicolumn{2}{|l|}{$\mathrm{BCa}$} & \multicolumn{2}{|l|}{ Boot-p } & \multicolumn{2}{|l|}{$\mathrm{BCa}$} \\
\hline & & Ave.Len & Cov.Pr & Ave.Len & Cov.Pr & Ave.Len & Cov.Pr & Ave.Len & Cov.Pr \\
\hline & & . & . & . & . & . & . & . & . \\
\hline \multirow{5}{*}{$\begin{array}{l}1 \\
5\end{array}$} & 5 & 1.6688 & 0.885 & 2.1491 & 0.931 & 3.4721 & 0.903 & 4.1228 & 0.922 \\
\hline & & & & & & & & & \\
\hline & 8 & 1.3832 & 0.925 & 1.5744 & 0.941 & 2.7090 & 0.913 & 3.1078 & 0.940 \\
\hline & 1 & 1.1283 & 0.928 & 1.2157 & 0.947 & 2.2591 & 0.921 & 2.4387 & 0.956 \\
\hline & 2 & & & & & & & & \\
\hline \multirow{6}{*}{$\begin{array}{l}2 \\
0\end{array}$} & 5 & 1.7373 & 0.903 & 2.0930 & 0.914 & 3.468 & 0.885 & 3.9722 & 0.939 \\
\hline & \multicolumn{9}{|c|}{ ) } \\
\hline & 1 & 1.2212 & 0.904 & 1.4162 & 0.938 & 2.4356 & 0.917 & 2.7846 & 0.941 \\
\hline & 0 & & & & & & & & \\
\hline & 1 & 1.0084 & 0.931 & 1.0886 & 0.949 & 2.0144 & 0.929 & 2.1712 & 0.948 \\
\hline & 5 & & & & & & & & \\
\hline 3 & 1 & 1.2319 & 0.925 & 1.4277 & 0.935 & 2.4380 & 0.917 & 2.9058 & 0.945 \\
\hline \multirow[t]{5}{*}{0} & 0 & & & & & & & & \\
\hline & 1 & 1.0061 & 0.940 & 1.1017 & 0.947 & 2.0134 & 0.927 & 2.2012 & 0.949 \\
\hline & 5 & & & & & & & & \\
\hline & 2 & 0.8740 & 0.942 & 0.9227 & 0.951 & 1.7485 & 0.927 & 1.8850 & 0.954 \\
\hline & 0 & & & & & & & & \\
\hline 4 & 1 & 1.0072 & 0.917 & 1.1396 & 0.940 & 2.0150 & 0.931 & 2.2548 & 0.932 \\
\hline \multirow[t]{5}{*}{0} & 5 & & & & & & & & \\
\hline & 2 & 0.8774 & 0.936 & 0.9355 & 0.944 & 1.7246 & 0.933 & 1.8955 & 0.943 \\
\hline & 0 & & & & & & & & \\
\hline & 3 & 0.7115 & 0.938 & 0.7454 & 0.954 & 1.4301 & 0.941 & 1.5090 & 0.951 \\
\hline & 0 & & & & & & & & \\
\hline 5 & 1 & 1.0049 & 0.926 & 1.5131 & 0.936 & 1.9937 & 0.924 & 2.2955 & 0.936 \\
\hline \multirow[t]{5}{*}{0} & 5 & & & & & & & & \\
\hline & 2 & 0.8806 & 0.933 & 0.9532 & 0.943 & 1.7485 & 0.932 & 1.9130 & 0.944 \\
\hline & 0 & & & & & & & & \\
\hline & 3 & 0.7108 & 0.942 & 0.7449 & 0.947 & 1.3842 & 0.943 & 1.5117 & 0.948 \\
\hline & 0 & & & & & & & & \\
\hline
\end{tabular}




\section{CONCLUSIONS}

In this paper we have proposed different procedures for estimating the exponential distribution parameter under Type-II censoring when the lifetime observations are fuzzy numbers. We have derived the MLE of the unknown parameter $\theta$. We have also introduced a computational approach method for estimating $\theta$ from fuzzy numbers. We have presented two procedures for constructing confidence intervals for $\theta$. We have then carried out a simulation study to assess the performance of all these procedures. Based on the results of this simulation study, we see clearly that, as the effective sample size increases, the performances of the MLEs in terms of MSEs become better. Also the performance of the estimates of $\theta$ based on the CAE method is as good as the MLEs. Hence, we recommend to use the CAE method for estimating $\theta$ from fuzzy data since it offers computational feasibility and also performs well in terms of MSEs. We also see that, compared to the ordinary percentile bootstrap confidence intervals, the $\mathrm{BCa}$ percentile bootstrap confidence intervals perform better in terms of the coverage probabilities although the confidence lengths are slightly larger. The coverage probabilities of the CIs based on the $\mathrm{BCa}$ percentile bootstrap method are quite close to the nominal level unless the effective relative sample fraction $\left(\frac{r}{n}\right)$ is small.

\section{REFERENCES}

[1] Arefi, M. and Taheri, S. M. (2011). Testing fuzzy hypothesis using fuzzy data based on fuzzy test statistic, Journal of uncertain systems. Vol. 5, No. 1, 45-61.

[2] Balakrishnan, N. and Cutler, C.D. (1995). Maximum likelihood estimation of Laplace parameters based on Type-II censored samples, In Statistical Theory and Applications: Papers in Honor of Herbert A. David (Eds. H.N. Nagaraja, P.K. Sen and D.F. Morrison). pp. 145-151, Springer-Verlag, New York.

[3] Balakrishnan, N. and Chan, P.S. (1992). Estimation for the scaled half logistic distribution under Type II. Computational Statistics and Data Analysis, Volume 13, Issue 2, 123-141.

[4] Balakrishnan, N. and Han, D. (2008). Exact inference for a simple step-stress model with competing risks for failure from exponential distribution under Type-II censoring. Journal of Statistical Planning and Inference, Volume 138, Issue 12, 172-4186. 
[5] Chan, P.S., Ng, H.K.T., Balakrishnan, N. and Zhou, Q. (2008). Point and interval estimation for extreme-value regression model under Type-II censoring. Computational Statistics and Data Analysis, Volume 52, Issue 8, 15, 4040-4058.

[6] Childs. A. and Balakrishnan. N. (1997). Maximum likelihood estimation of Laplace parameters based on general Type-II censored samples. Statistical Papers, 38, 343-349.

[7] Coppi, R., Gil, M.A. and Kiers, H.A.L., (2006). The fuzzy approach to statistical analysis. Computational Statistics and Data Analysis, 51(1), 1â€"“14.

[8] Dallas, R. W. (1993). Maximum likelihood estimation of Burr XII distribution parameters under Type II censoring. Microelectronics Reliability, Volume 33, Issue 9, 1251-1257.

[9] Denoeux, T. (2011). Maximum likelihood estimation from fuzzy data using the EM algorithm, Fuzzy Sets and Systems. doi:10.1016/j.fss.2011.05.022

[10] Dubois, D. and Prade, H.(1980). Fuzzy Sets and Systems: Theory and Applications. Academic Press, New York.

[11] Efron, B.(1982). The Jackknife, the bootstrap and other resampling plans. In: CBMS-NSF Regional Conference Series in Applied Mathematics 38, SIAM, Philadelphia, PA. [12] Efron, B. and Tibshirani R.(1982). An introduction to the bootstrap. Chapman and Hall, New York.

[13] Gebhardt, J., Gil M.A. and Kruse R., (1998). Fuzzy set-theoretic methods in statistics, in:R.Slowinski(Ed.), Fuzzy Sets in Decision Analysis, Operations Research and Statistics, Kluwer Academic Publishers, Boston, pp.311-347.

[14] Grzegorzewski, P. and Hryniewicz O.,(2002). Computing with words and life data, International Journal of Applied Mathematics and Computer Science, Vol. 12, No.3, 337-345. [15] Huang, H., Zuo, M. and Sun, Z., (2006). Bayesian reliability analysis for fuzzy lifetime data. Fuzzy Sets and Systems, 157, 1674ât'“1686.

[16] Iliopoulos, G.and Balakrishnan, N.,(2011). Exact likelihood inference for Laplace distribution based on Type-II censored samples, Journal of Statistical Planning and Inference, Volume 141, Issue 3, 1224-1239.

[17] Lawless, J. F.(2003). Statistical Models and Methods for Lifetime Data, 2nd Edition, John Wiley \& Sons, New Jersey. 
[18] Ng, H.K.T., Kundu, D. and Balakrishnan N.,(2006). Point and interval estimation for the two-parameter Birnbaum-Saunders distribution based on Type-II censored samples, Computational Statistics and Data Analysis, Volume 50, Issue 11, 20, 3222-3242

[19] A., Pak, G.H., Parham, and M., Saraj, (2013). Inference for the Weibull Distribution Based on Fuzzy Data. Revista Colombiana de Estadistica, 36(2), 339-358.

[20] A., Pak, G.H., Parham, and M., Saraj, (2014). Inferences on the Competing Risk Reliability Problem for Exponential Distribution Based on Fuzzy Data. IEEE Transactions on Reliability, 63(1), 1-10.

[21] A., Pak, G.H., Parham, and M., Saraj, (2014). Inference for the Rayleigh Distribution Based on Progressive Type-II Fuzzy Censored Data. Journal of Modern Applied Statistical Methods, 13(1), 287-304.

[22] Panahi, H. and Asadi, S.(2011). Estimation of the Weibull Distribution Based on Type-II Censored Samples, Applied Mathematical Sciences, Vol. 5, no. 52, 2549-2558

[23] Pandey, B.N., Malik, H.J. and Srivastava, R. (1989). Shrinkage estimators for the shape parameter of Weibull distribution under Type-II censoring, Communications in Statistics-Theory and Methods, 18, 1175-1199.

[24] Schneider, H. and Weissfeld L. (1986). Inference based on type II censored samples. Biometrics, 1986 Sep;42(3):531-6.

[25] Singh, U., Gupta, P. K. and Upadhyay S.K. (2005). Estimation of parameters for exponentiated-Weibull family under type-II censoring scheme, Computational Statistics \& Data Analysis, 48 , 509-523.

[26] Zadeh, L. A. (1968). Probability measures of fuzzy events, Journal of Mathematical Analysis and Applications10, 421-427.

[27] Zimmermann, H. J. (1991). Fuzzy set teory and its application, Kluwer, Dordrecht.

\section{How to cite this article:}

Makhdoom I, Nasiri P. Maximum likelihood estimation of exponential distribution under type-ii censoring from imprecise data. J. Fundam. Appl. Sci., 2016, 8(2S), 697-714. 